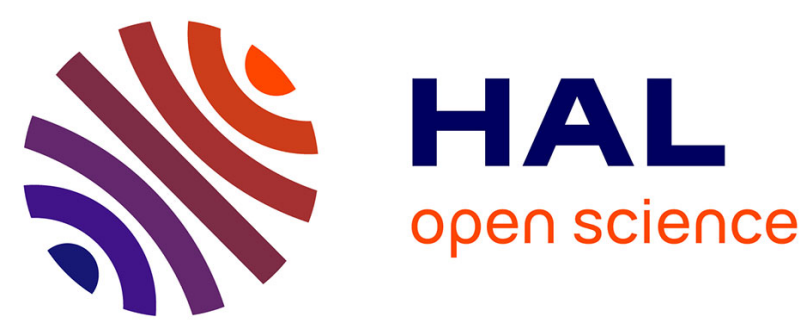

\title{
An Accurate Cooperative Positioning System for Vehicular Safety Applications
}

João Pinto Neto, Lucas Gomes, Fernando Ortiz, Thales Almeida, Miguel Elias Mitre Campista, Luis Henrique Maciel Kosmalski Costa, Nathalie Mitton

\section{- To cite this version:}

João Pinto Neto, Lucas Gomes, Fernando Ortiz, Thales Almeida, Miguel Elias Mitre Campista, et al.. An Accurate Cooperative Positioning System for Vehicular Safety Applications. Computers and Electrical Engineering, 2020, 83, 10.1016/j.compeleceng.2020.106591 . hal-02364355

\section{HAL Id: hal-02364355}

\section{https://hal.inria.fr/hal-02364355}

Submitted on 14 Nov 2019

HAL is a multi-disciplinary open access archive for the deposit and dissemination of scientific research documents, whether they are published or not. The documents may come from teaching and research institutions in France or abroad, or from public or private research centers.
L'archive ouverte pluridisciplinaire HAL, est destinée au dépôt et à la diffusion de documents scientifiques de niveau recherche, publiés ou non, émanant des établissements d'enseignement et de recherche français ou étrangers, des laboratoires publics ou privés. 


\title{
An Accurate Cooperative Positioning System for Vehicular Safety Applications
}

João B. Pinto Neto ${ }^{1}$, Lucas C. Gomes ${ }^{1}$, Fernando M. Ortiz ${ }^{1}$, Thales T. Almeida $^{1}$, Miguel Elias M. Campista ${ }^{1}$, Luís Henrique M. K. Costa $^{1}$, Nathalie Mitton $^{2}$

\begin{abstract}
Typical Global Navigation Satellite System (GNSS) receivers offer precision in the order of meters. This error margin is excessive for vehicular safety applications, such as forward collision warning, autonomous intersection management, or hard braking sensing. In this work we develop CooPS, a GNSS positioning system that uses Vehicle to Vehicle (V2V) and Vehicle to Infrastructure (V2I) communications to cooperatively determine absolute and relative position of the ego-vehicle with enough precision. To that end, we use differential GNSS through position vector differencing to acquire track and across-track axes projections, employing elliptical and spherical geometries. We evaluate CooPS performance by carrying out real experiments using off-the-shelf IEEE 802.11p equipment at the campus of the Federal University of Rio de Janeiro. We obtain an accuracy level under 1.0 and $1.5 \mathrm{~m}$ for track (where-in-lane) and across-track (which-lane) axes, respectively. These accuracy levels were achieved using a $2.5 \mathrm{~m}$ accuracy circular error probable (CEP) of $50 \%$ and a $5 \mathrm{~Hz}$ navigation update rate GNSS receiver.

Keywords: Cooperative GNSS positioning, DSRC, IEEE 802.11p.

\footnotetext{
Email addresses: pinto@gta.ufrj.br (João B. Pinto Neto), gomes@gta.ufrj.br (Lucas

${ }^{1}$ GTA/PEE-COPPE/DEL-Poli, Federal University of Rio de Janeiro, Brazil.

${ }^{2}$ National Institute for Research in Computer Science and Automatic Control, Lille, France.
} C. Gomes), fmolano@gta.ufrj.br (Fernando M. Ortiz), almeida@gta.ufrj.br (Thales T. Almeida), miguel@gta.ufrj.br (Miguel Elias M. Campista), luish@gta.ufrj.br (Luís Henrique M. K. Costa), mitton@inria.fr (Nathalie Mitton)
\end{abstract}




\section{Introduction}

Vehicular communications support the development of various applications of Intelligent Transportation Systems (ITS), from infotainment to traffic safety. In this paper we focus on safe driving applications, most of which rely on information about location, speed, and direction of vehicles. That information is often obtained from Global Navigation Satellite System (GNSS) receivers. Vehicular safety applications, in addition to helping accident prevention, increase traffic law compliance, improve incident management, and facilitate crash investigations. Other than safe driving, applications which benefit from accurate vehicle positioning include autonomous vehicles, entertainment, traffic light control, assisted driving and detection of accidents 1, 2, 3, 4, 5, 6. Vehicle safety applications specifically require accurate positioning systems to improve vehicular navigation. This is the case of lane-level positioning and collision avoidance systems [7, 8, 9, 10. Nevertheless, the accuracy of GNSS receivers is often not enough. Moreover, it is compromised in urban canyons and under foliage by multipath, non-line-of-sight or complete blockage of GNSS signals [11.

Positioning systems based on GNSS are typically prone to errors in the order of meters, which exceed the acceptable maximum for various safe driving applications. For example, in a forward collision warning application, errors of this magnitude increase the risk of accidents, specially at higher speeds [12, 13. As another example, considering that streets and road lanes have widths between 2.5 and $3.5 \mathrm{~m}$, a lane-level positioning system using only an autonomous single carrier (L1) GNSS receiver would be unreliable since errors and lane widths are in the same order of magnitude. Even though GNSS techniques such as DGNSS (Differential Global Navigation Satellite System), PPP (Precise Point Positioning), or RTK (Real Time Kinematics) respectively provide meter, centimeter, and millimeter accuracy, their performance is also affected by the number of visible satellites and by multipath propagation [14]. One way of circumventing these issues is to deploy a positioning system based on multiple inputs coming from collaborative sources, to compensate for individual errors. 
Those collaborative sources include Vehicle to Vehicle (V2V) and Vehicle to Infrastructure (V2I) communications, in-vehicle sensors, Light Detection and Ranging (LIDAR), cameras, and digital maps [15, 16, 17, 18. Typically, vehicular positioning solutions use subsets of those inputs. Thus, the number of devices to coordinate and the costs involved vary.

Positioning accuracy for vehicle safety is classified into three distinct levels: which-road $(5.0 \mathrm{~m})$, which-lane $(1.5 \mathrm{~m})$ and where-in-lane (under $1.0 \mathrm{~m})$. The first means only to assess if the vehicles involved are on the same road. The second level of accuracy enables a vehicle to identify other vehicles that are traveling on the same or adjacent lanes, while the third level allows the identification of the vehicle's position inside the lane. Electronic Emergency Brake Light (EEBL), Forward Collision Warning (FCW), and Lane Change Advisor (LCA) are examples of vehicle safety applications that require the three accuracy levels, respectively [19].

In this paper we propose Cooperative GNSS Positioning System (CooPS), a system designed to provide which-lane accuracy. To obtain this accuracy level, CooPS uses (i) a combination of V2V and V2I communications over the Dedicated Short Range Communications (DSRC) band in a cooperative way, (ii) the well-known differential GNSS through the position vector differencing method, 50 (iii) a novel technique to compute track and across track axes projections, and (iv) the assumption that GPS receivers located in the same road stretch share the same satellite constellation and ephemerids to overcome the low accuracy (of 10.0 m, typically) 20] of L1 GNSS receivers in Single Point Positioning (SPP) mode. One design requirement is to achieve accurate driving using only off-theshelf GNSS receivers and, as a consequence, avoid compatibility issues imposed by additional sensors between vehicular equipment and the embedded GNSS. The use of fewer sensors reduces direct and indirect costs, like additional electrical wiring. Thus, another design goal is to use as few as possible data sources. We evaluate CooPS performance by carrying out experiments at the campus 60 of Federal University of Rio de Janeiro (UFRJ) using IEEE 802.11p devices, installed along the roadway and inside the vehicle. The system is validated us- 
ing the real distance between RSUs and Google Earth projections. The results show that CooPS achieves where-in-lane positioning accuracy with respect to cross-track axis and which-lane with respect to track axis using only a GNSS receiver as positioning input. As such, CooPS provides a low-cost solution, and in addition operates in any vehicle regardless of brand, cost or age.

This work is organized as follows. Section 2 positions CooPS with respect to related work. Section 3 introduces CooPS and provides an analysis of GNSS error sources. Section 4 details the CooPS proposal, the empirical methodology, and the geometric model considered. Field experiments are described in Section 5, as well as the results obtained in a real scenario, which serves as the proof of concept of CooPS. Finally, Section 6 provides closing remarks and discusses future work.

\section{Related Work}

In this section, we focus on related works similar to the proposed system, i.e., designed to achieve where-in-lane level for navigation and collision warning applications, using multiple sensors or cooperative approaches.

Different positioning systems using multiple data sources have been investigated in the literature. The sources of information can be digital maps, GNSS, Inertial Measurement Units (IMU) and data acquired directly from the CAN bus of the vehicle [21. Tsai et al. 22] propose IPC (Improving Positioning in real City environments), a cooperative system that combines an autonomous GPS and a camera to improve the accuracy of relative positioning in urban environments. IPC runs an algorithm that uses V2V communications in addition to the GPS and camera, to determine the position of the vehicle relative to its neighbors. In case of GPS failure, IPC relies only on the camera and V2V communications to perform navigation. Conversely, if the camera fails, GPS is used, performing mutual compensation between the navigation modes. IPC is a complete solution for relative positioning, nonetheless, it relies on the existence of a camera, V2V communications, and a GPS. CooPS on the other hand relies 
only on V2V and V2I communications, and a GPS to achieve accuracy below $1.5 \mathrm{~m}$. Even considering that an additional camera does not add much complexity to the system, the reduction of $15 \%$ with respect to the raw GPS positioning error achieved by IPC yields an error greater than $4 \mathrm{~m}$. This performance does 95 not meet the requirements of vehicle safety applications.

Ansari et al. 23. propose a cooperative network architecture that is used to distribute differential corrections using the standard Radio Technical Commission for Maritime Services (RTCM) message format [24] and V2I communications. The Road Side Unit (RSU) receives geographic positions from an embedded GPS receiver, compatible with the RTK technique, and performs corrections using the data received from the nearest Continuous Operating Reference Station (CORS). The communications between the CORS and the RSU goes through the $3 \mathrm{G}$ cellular network that carries the correction messages using the Networked Transport of RTCM via Internet Protocol (NTRIP) [25]. This data is received at the OBUs also using the NTRIP protocol, which, in turn, allows the correction of the OBUs positions. The authors call this architecture Real-time Relative Positioning (RRP) and claim that it guarantees relative positioning of vehicles with centimeter precision, according to the experimental analysis carried out against various traffic scenarios. The proposal presents an accurate positioning system which meets the requirements of vehicle safety applications. Nevertheless, the system cost is high, due to the RTK GPS equipment and the need for a permanent communication with a CORS. In contrast, CooPS does not require permanent connection with a CORS and uses an off-the-shelf GNSS receiver.

Roth et al. 26] propose a collaborative positioning system also designed to reduce the number of sensors. They use an autonomous single carrier GPS installed in each vehicle as the only positioning sensor, and V2V communications to perform vehicle self-localization. The distance between each satellite and the Earth (pseudoranges) received by the vehicles in range are shared and, in case 120 a vehicle's GPS receiver fails due to lack of satellite availability, neighboring vehicles act as sources of satellite data. The proposed Advanced Shared Pseudo- 
range Algorithm (ASP) uses a least squares position estimation and the shared information to improve positioning accuracy, mitigating the problem of satellite unavailability in urban environments. ASP shares with CooPS low hardware cost and high degree of compatibility. Nevertheless, the accuracy achieved by ASP does not meet the requirement of vehicle safety applications, the position error ranging from 10 to $15 \mathrm{~m}$.

Huang and Lin 27. propose a collision warning system based on three inputs: speed variation, direction change, and position interruption. The latter is defined as the time the system stays in the same position, which is equal to the GPS update period, for practical reasons. The proposed Vector Cooperative Collision Warning (VCCW) system evaluates the collision risk by considering a vehicle and all of its neighbors within the same coverage area, once per second. If there is a collision risk, a subsystem computes the safe braking distance and the time needed to reach this distance. The simulation of VCCW has shown safe braking distance errors below $3 \mathrm{~cm}$. The work improves collision warning algorithms by also considering speed and direction variations, and position interruption over time. To accomplish that, VCCW takes account of the acceleration of the vehicle and the uses a vector-based algorithm to avoid collision even if vehicles change the course in a curve. Moreover, VCCW compensates for the time between two GPS acquisitions by adding the estimated distance traveled by the vehicle to its position. It also increases the accuracy with respect to errors introduced by the GNSS update rate. The performance of VCCW meets the requirements of vehicle safety applications: it is one of the few works that tackle the GNSS update rate issue. The main difference to the present work is that CooPS uses a novel geometric model, based on empirical results, to estimate the relative distance between vehicles. CooPS is simpler because it does not require coordinates transformations. Furthermore, compared with VCCW, which directly computes the distances between vehicles based on their GPS coordinates, CooPS provides a better accuracy by using an external reference (the RSU location). CooPS uses V2V and V2I communications, instead of only V2V, as VCCW does. We validate our proposal through real experiments. 


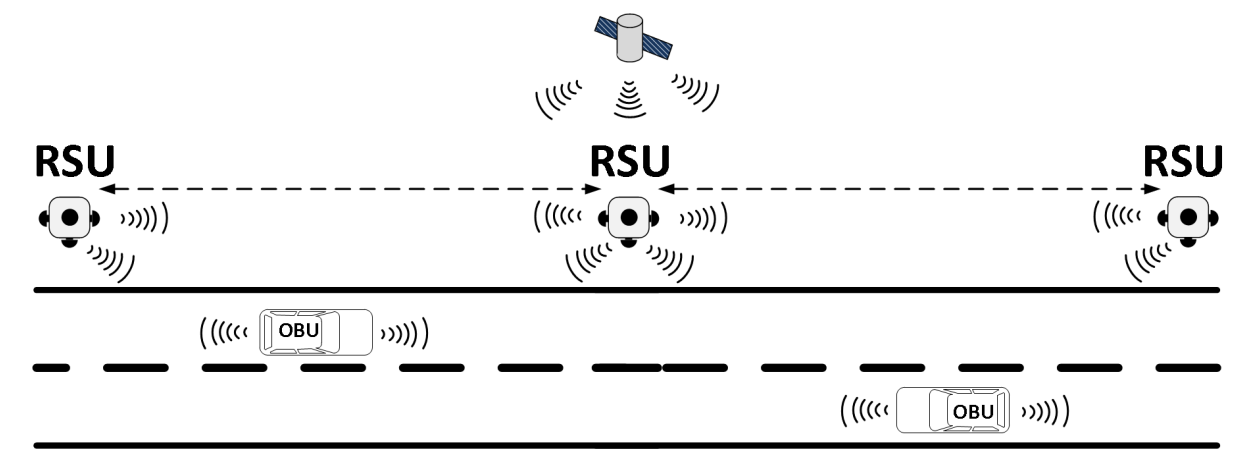

Figure 1: Application scenario of CooPS where the Road Side Units, deployed along the road, broadcast their ground truth geographic position to the On-Board Units. This location information is used by the system embedded in the OBUs to determine the relative distance between vehicles.

VCCW reports an error lower than $3 \mathrm{~cm}$, but only for the determination of the safe braking distance, and only in simulations. The error was not measured in the experimental prototype; that is why it is shown as "not available" in Table 1.

Although most of the systems described above provide accuracy levels that meet where-in-lane requirements, their deployment is mainly relevant when GNSS fails to provide a reliable position. Thus, the main difference compared with CooPS is that it uses only the GNSS receiver as a positioning device, in the same conditions where other systems need additional sensors to achieve wherein-lane accuracy level. On the other hand, CooPS depends on a GNSS system and then, whenever it fails, e.g., when the vehicle enters a canyon, tunnel, or dense forest, the navigation may face interruptions. In this case, dead reckoning positioning techniques [28, 29] can be used. Dead reckoning techniques do not require additional sensors and can operate using only data from available built-in sensors, such as wheel speed or steering angle sensors. Table 1 provides a brief comparison between CooPS and previous proposals of the literature. 
Table 1: Comparison between CooPS and related work.

\begin{tabular}{|c|c|c|c|c|c|}
\hline Proposal & Error & Hardware & Network & $\begin{array}{c}\text { Dead } \\
\text { Reckoning }\end{array}$ & Cost \\
\hline CooPS & $<1.5 \mathrm{~m}$ & $\begin{array}{c}\text { GNSS, } \\
\text { OBUs } \\
\text { and RSUs }\end{array}$ & $\begin{array}{c}\text { V2V } \\
\text { and V2I }\end{array}$ & No & Low \\
\hline IPC [22] & $>4 \mathrm{~m}$ & $\begin{array}{c}\text { GNSS, } \\
\text { camera } \\
\text { and OBUs }\end{array}$ & V2V & Yes & Medium \\
\hline RRP [23] & Centimetric & $\begin{array}{c}\text { RTK GPS, } \\
\text { CORS } \\
\text { station }\end{array}$ & $3 \mathrm{G}$ & No & High \\
\hline ASP [26] & $<15 \mathrm{~m}$ & $\begin{array}{c}\text { GNSS and } \\
\text { OBUs }\end{array}$ & $3 \mathrm{G}$ & Yes & Low \\
\hline VCCW [27 & N/A & $\begin{array}{c}\text { GNSS, ac- } \\
\text { celerometer } \\
\text { and OBUs }\end{array}$ & V2V & Yes & Medium \\
\hline
\end{tabular}

\section{Accurate Positioning Problem for Safe Driving Applications}

After receiving information from the RSUs using V2I communication, the application running in the OBU is able to find the ego-vehicle localization and, 
furthermore, compute the relative and absolute position in the current road over long time intervals in the order of hours. They also conclude that, if two GPS receivers are close enough (less than some tens of $\mathrm{km}$ ), the errors caused 
by the effect of the ionosphere and of the troposphere are highly correlated. Under this condition, the differential error of GPS receivers related to the ionosphere and the troposphere is very small (under $1 \mathrm{~m}$ ). Considering the vehicular communication scenario, a driving safety application is typically concerned with events that occur in seconds or at most a few minutes. Moreover, the distances between RSUs should be less than $1 \mathrm{~km}$, the theoretical radio communication range of IEEE $802.11 \mathrm{p}$ wireless devices 31, and around $300 \mathrm{~m}$ range for a better communication performance 32. Hence, we assume that the relevant GPS error sources are multipath propagation and receiver noise, for the application scenario of vehicle safety applications.

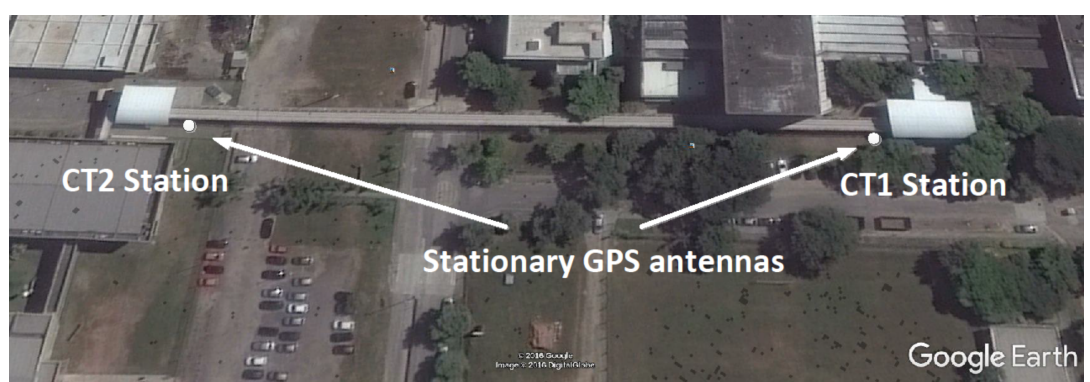

Figure 2: Aerial view of the first experimentation site: CT1 and CT2 Stations.

\subsection{Experiments using GPS receivers in SPP mode}

Our first empirical experiments at the campus of UFRJ confirm that obtaining sub-metric positioning errors using GPS receivers operating in SPP mode is a challenge. We collect and analyze data from two GPS stationary stations separated by 160 meters, shown in Fig. 2 , one located at the Technology Center 1 (CT1 Station) and another at the Technology Center 2 (CT2 Station) ${ }^{3}$ CT1 and CT2 Stations have single carrier (L1) autonomous GPS receivers with an accuracy of $2.5 \mathrm{~m} \mathrm{CEP}$ in $50 \%$ of the measurements taken in a time interval of 24 hours. Measurements were taken at both stations at one sample per second

\footnotetext{
${ }^{3}$ Those are the stations of MagLev, the magnetic levitation train prototype developed at COPPE/UFRJ. CooPS will be used in the future to automate the train braking system.
} 


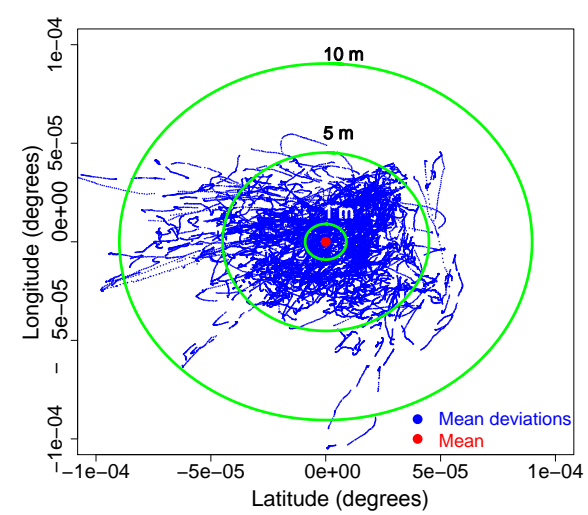

(a) CT1 Station.

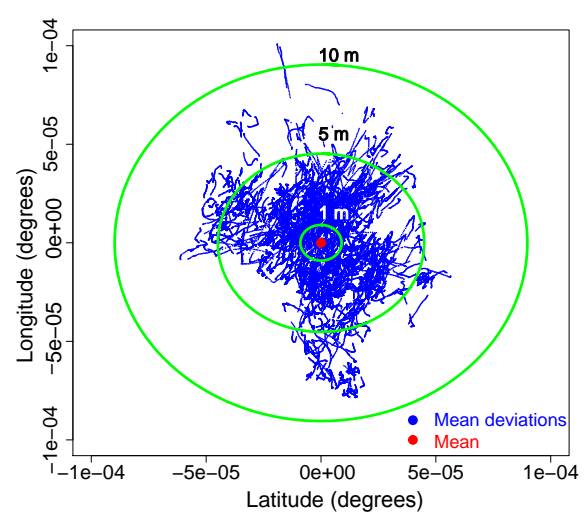

(b) CT2 Station.

Figure 3: Mean deviations (errors) of coordinates acquired from GPS receiver stations during a 24-hour period. Green circles enclose deviations smaller than 1, 5, or 10 meters, respectively.

rate, during $24 \mathrm{~h}$. Figs. $3 \mathrm{a}$ and $3 \mathrm{~b}$ show the differences of acquired geographical coordinates (blue dots), in degrees from their mean (full red dot), denoted herein by deviations. The inner, intermediate and outer green circles represent boundaries corresponding respectively to the distances of 1,5 and $10 \mathrm{~m}$ from the mean. We can observe that deviations greater than $10 \mathrm{~m}$ from the mean are more frequent at CT1 Station, which is in proximity of tall buildings (Fig. 2). This increases multipath reception errors and reduces the number of visible satellites.

The deviations shown in Figs. 3a and 3b confirm the hypothesis that GPS receivers working in SPP mode do not meet the requirements of vehicle safety applications. Nevertheless, such deviations occurred in a time interval of 24 hours, which is prone to all error sources described in Section 3.1 and they are all computed as the distance to a fixed coordinate reference. Nevertheless, vehicle safety applications are related to events that occur at short time intervals, at short distances between the vehicles and within the same environment. Thus, we have set a maximum time interval between measures of $90 \mathrm{~s}$ (which corresponds approximately to a distance of $1400 \mathrm{~m}$ for a vehicle at $60 \mathrm{~km} / \mathrm{h}$ ) to 


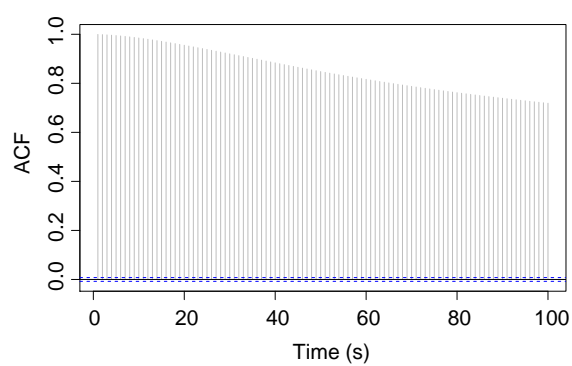

(a) CT1 Station.

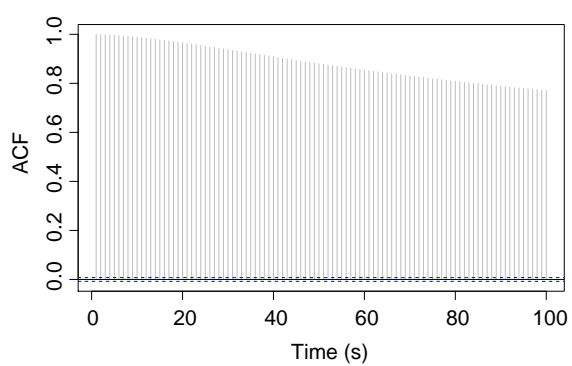

(b) CT2 Station.

Figure 4: Time correlation of deviations acquired by the static GPS station receivers from a fixed coordinate at $90 \mathrm{~s}$ interval calculated using autocorrelation function (ACF) of time series of the errors.

evaluate time correlation of the deviations (errors). As the data was acquired at 1 s sample period, applying a proper operator we convert it to a time series and calculate the corresponding autocorrelation function (ACF) depicted in Fig. 4. As the figure shows, the deviations of both stations are highly correlated, which confirm the well-known behavior of static GPS receivers. As expected, a better time correlation for CT2 (Fig. 4b) station than CT1 station (Fig. 4a) can be observed due to different multipath conditions. To evaluate the spatial correlation, we consider the difference between consecutive measurements instead of the absolute value between the samples and its 24 -hour mean. This is equivalent to the use of the last measurement as reference coordinate, which reduces the deviation as consecutive measurements are expected to vary more smoothly. Let $p_{0}, p_{1}, \ldots, p_{n}$ be a sequence of coordinates acquired from a GPS receiver at a fixed rate and $d_{0}, d_{1}, \ldots, d_{n}$ the distances from these points to a fixed reference coordinate $p_{\text {ref }}$. Denoting $\tau$ a predefined time interval and $\epsilon_{k}$ as the error of the distances measured at time $t_{k}$ and $t_{k+\tau}$, we have:

$$
\epsilon_{k}=d_{k+\tau}-d_{k}
$$

Thus, considering $0 \leq \tau \leq 90 s, \epsilon_{k}$ is mainly produced by multipath carrier effect whose behavior we are interested to figure out if it meets the requirements of 


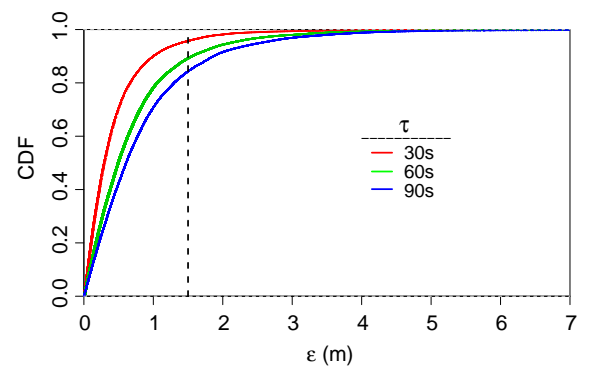

(a) CT1 station.

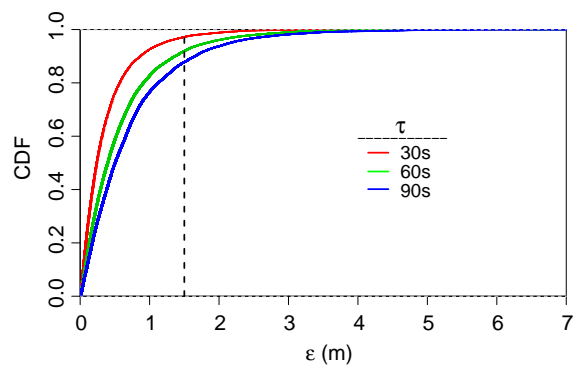

(b) CT2 station.

Figure 5: Cumulative density function (CDF) of $\epsilon$ to evaluate spatial correlation of multipath effect. The vertical dashed line at $1.5 \mathrm{~m}$ establish the maximum admitted value to meet vehicle safety applications requirements.

vehicle safety applications. For most applications, which-lane accuracy $(1.5 \mathrm{~m})$ is required. Analyzing the cumulative distribution function (CDF) of $\epsilon$ (Fig. 5) one can note that the $1.5 \mathrm{~m}$ threshold is achieved by more than $80 \%$ of the samples for CT1 station and almost $90 \%$ for CT2 station even for $\tau=90 \mathrm{~s}$. Although these results are valid for static receivers, our proposition extends this concept to connected vehicles environment taking into account differential GPS through position vector differencing between a surveyed-coordinates RSU (static base) and an OBU (moving base), sharing the same satellite constellation and ephemerids. Our goal is to achieve which-lane positioning accuracy to meet the requirements of mostly vehicle safety applications within a dynamic window of $90 \mathrm{~s}$.

\section{The Proposed Cooperative GNSS Positioning System}

The main goal of CooPS (Cooperative GNSS Positioning System) is to provide at least which-lane accurate positioning, required by vehicle safety applications. CooPS adopts differential GPS through position vector differencing within the window of time in which vehicular security events occur. This simple difference of coordinates allows CooPS to operate with any GPS receiver 
device that just provides latitude, longitude, and speed information. The determination of the relative positions between vehicles and the absolute position of the vehicle itself within the stretch delimited by the RSUs is carried out using the projections of the vectors on the track and across-track axes, from now on denoted by road and lane axes. These projections are calculated using a new method based on elliptical and spherical geometry, shown ahead.

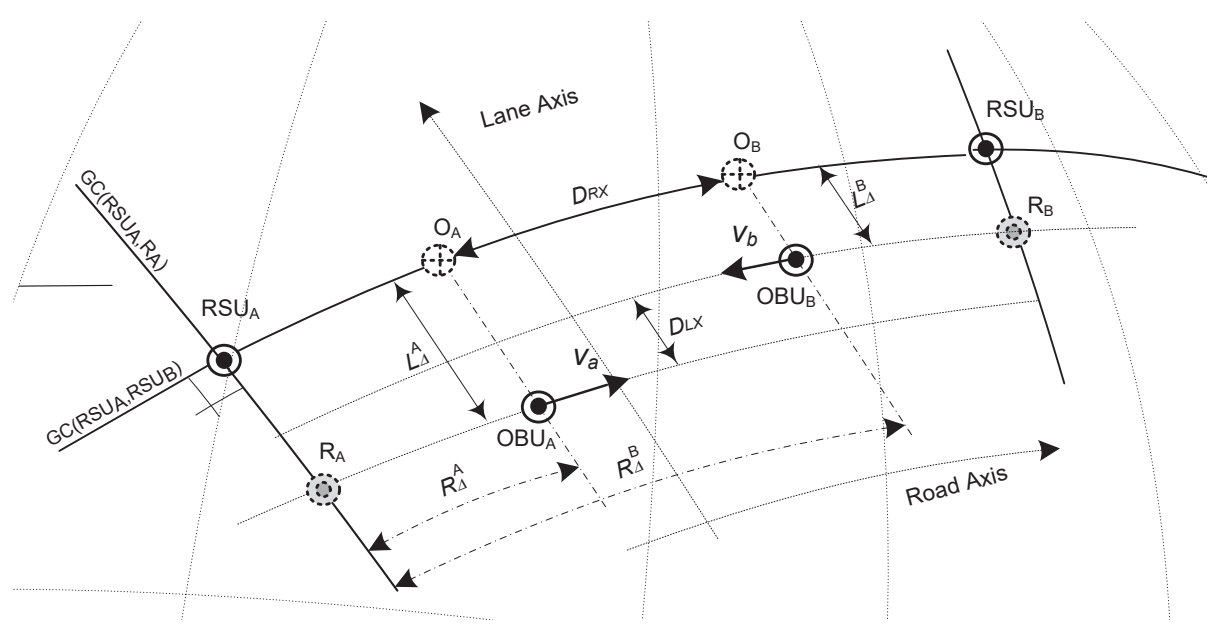

Figure 6: Geometric model used by CooPS to determine the relative distances $D_{R X}$ and $D_{L X}$ between the OBUs with respect to road and lane axes, respectively. $O B U_{A}$ and $O B U_{B}$ represent vehicles traveling in different lanes at $v_{a}$ and $v_{b}$ speeds, respectively. $O B U_{A}$ and $O B U_{B}$ are in the same road stretch, delimited by the road-side units $R S U_{A}$ and $R S U_{B}$. The $\operatorname{arcs} R_{\Delta}^{A}, R_{\Delta}^{B}, L_{\Delta}^{A}$, and $L_{\Delta}^{B}$ represent the road and lane axes displacement projections over their respective great circles (GCs).

Fig. 6 illustrates the projection of the straight line between $O B U_{A}$ and $R S U_{A}$ over the road, $R_{\Delta}^{A}$, and over the lane axis, $L_{\Delta}^{A}$. Similarly, the projection of the vector between $\mathrm{OBU}_{B}$ and $\mathrm{RSU}_{B}$ produces the vectors $R_{\Delta}^{B}$ and $L_{\Delta}^{B}$ over the road and lane axis, respectively. Hence, considering that all vehicles use the same coordinate system, they only need to share its great circle projections ( $R_{\Delta}$ to calculate the relative distance and $L_{\Delta}$ ), therefore detecting a potential collision which would occur if the vehicles have the same $L_{\Delta}$ ) value. CooPS geometric model uses the known surveyed positions of $R S U_{A}$ and $R S U_{B}$ to 
estimate the values of $R_{\Delta}$ and $L_{\Delta}$. Therefore, besides calculating, for each new position received, the relative distance of the vehicles, CooPS also estimates their absolute position within the road segment. This feature is very useful for V2I safety applications.

As for the coordinate system, CooPS is based on the datum World Geodesic System 1984 (WGS84) reference ellipsoid to compute long distances for geographic coordinates (notation: $\phi=$ Latitude, $\lambda=$ Longitude). For some specific functions which operate over short distances, we use the spherical model in CooPS. In Fig. 6, points $R S U_{A}$ and $R S U_{B}$ represent two consecutive Road Side Units, located at geographic coordinates $\left(\phi_{R S U_{A}}, \lambda_{R S U_{A}}\right)$ and $\left(\phi_{R S U_{B}}\right.$, $\left.\lambda_{R S U_{B}}\right)$, respectively. $O B U_{A}$ and $O B U_{B}$ points represent the OBUs carried by two vehicles, currently located at the geographic coordinates $\left(\phi_{O B U_{A}}, \lambda_{O B U_{A}}\right)$ and $\left(\phi_{O B U_{B}}, \lambda_{O B U_{B}}\right)$, respectively. Note that the coordinates of the vehicles are informed by the GPS receivers of the OBUs. $O B U_{A}$ and $O B U_{B}$ travel at speeds $v_{a}$ and $v_{b}$, respectively. The road axis, parallel to the great circle (GC) formed by $R S U_{A}$ and $R S U_{B}$, is used to determine $D_{R X}$, the relative distance of vehicles regarding the traveling direction, whereas the lane axis is used to determine $D_{L X}$, the relative distance regarding to the lateral direction.

These relative distances can be calculated through the OBU's projections $O_{A}, O_{B}, R_{A}$, and $R_{B}$. Using V2I communications, the RSUs periodically send their ground-truth geographic coordinates to the OBUs. Rather than applying the simple difference to make positioning corrections, CooPS calculates the angular distance related to the projections of the acquired GPS positions over two orthogonal great circles (GCs), as represented in Fig. 6. These angular distances are used by CooPS to correct the OBU position in a simple and efficient way, avoiding the computational effort of coordinate transformations.

\subsection{CooPS Positioning Algorithm}

To obtain the distance between the two vehicles, CooPS first calculates the distances of the projections along the road axis defined by the GC that connects points $R S U_{A}$ and $R S U_{B}$, denoted as $\operatorname{GC}\left(R S U_{A}, R S U_{B}\right)$. Then, it executes the 
same procedure regarding the lane axis, defined by the GC which is orthogonal to the first one, denoted by $\operatorname{GC}\left(R S U_{A}, R_{A}\right)$. Considering $O B U_{A}$, the system calculates, for each position informed by the GPS, the angular distances $L_{\Delta}$ and $R_{\Delta}$ from the points $O B U_{A}$ and $O B U_{B}$ to the $\operatorname{GC}\left(R S U_{A}, R_{A}\right)$. As Fig. 6 illustrates, these distances are the same as the $\overline{R S U_{A} O_{A}}$ and $\overline{R S U_{B} O_{B}}$ projections of the given points over $\operatorname{GC}\left(R S U_{A}, R S U_{B}\right)$. Therefore, the road axis relative distance $D_{R X}$ between the OBUs is:

$$
D_{R X}=\left|R_{\Delta}^{A}-R_{\Delta}^{B}\right|
$$

Similarly, with respect to the lane axis, we calculate the angular distances $L_{\Delta}^{A}$ and $L_{\Delta}^{B}$ from the $O B U_{A}$ and $O B U_{B}$ points to the circle $\operatorname{GC}\left(R S U_{A}, R S U_{B}\right)$. Hence, the lane axis relative distance $D_{L X}$ between the OBUs is calculated as:

$$
D_{L X}=\left|L_{\Delta}^{A}-L_{\Delta}^{B}\right| .
$$

CooPS can also be used to determine the absolute position of the OBUs. The procedure is similar, except that there must be an external trigger, for example, a sensor on the vehicle, to establish a reference to correct the position with respect to both axes of the road.

CooPS assumes that there is a communication link between RSUs and OBUs along the road stretch and that the maximum distance between RSUs is smaller than the wireless network range. We only describe the procedure for determining the relative distance for $\mathrm{OBU}_{A}$, since it is identical for $\mathrm{OBU}_{B}$. Thus, three steps are performed before calculating $L_{\Delta}$ and $R_{\Delta}$ :

Step 1) Compute the initial Azimuth between $R S U_{A}$ and $R S U_{B}$.

Using the elliptical model implemented by Vincenty solution [33] enhanced by Karney [34, the initial azimuth (bearing) $\beta_{A B}$ from $R S U_{A}$ at $\left(\phi_{R S U_{A}}, \lambda_{R S U_{A}}\right)$ to $R S U_{B}$ at $\left(\phi_{R S U_{B}}, \lambda_{R S U_{B}}\right)$ can be computed as:

$$
\beta_{A B}=\arctan 2(a, b),
$$

where $a=\sin \left(\Delta_{\lambda}\right) \cdot \cos \left(\phi_{R S U_{B}}\right), b=\cos \left(\phi_{R S U_{A}}\right) \cdot \sin \left(\phi_{R S U_{B}}\right)-\sin \left(\phi_{R S U_{A}}\right)$. $\cos \left(\phi_{R S U_{B}}\right) \cdot \cos \left(\Delta_{\lambda}\right)$, and $\Delta_{\lambda}=\lambda_{R S U_{B}}-\lambda_{R S U_{A}}$. 
Step 2) Compute the initial Azimuth between $R S U_{A}$ and $O B U_{A}$.

Using Equation 4, the initial azimuth $\beta_{A A}$ is obtained from $R S U_{A}$ at $\left(\phi_{R S U_{A}}, \lambda_{R S U_{A}}\right)$

Step 3) Compute the distance between $R S U_{A}$ and $O B U_{A}$.

The angular distance $d_{A A}$ between points $R S U_{A}$ at $\left(\phi_{R S U_{A}}, \lambda_{R S U_{A}}\right)$ and $O B U_{A}$ at $\left(\phi_{O B U_{A}}, \lambda_{O B U_{A}}\right)$ can be obtained by using the Haversine formula [35], a spherical model, as:

$$
d_{A A}=2 \cdot \operatorname{atan} 2(\sqrt{c} / \sqrt{1-c}),
$$

where $c=\sin ^{2}(\Delta \phi / 2)+\cos \left(\phi_{R S U_{A}}\right) \cdot \cos \left(\phi_{O B U_{A}}\right) \cdot \sin ^{2}\left(\Delta_{\lambda} / 2\right), \Delta \phi=\phi_{R S U_{A}}-$ $\phi_{O B U_{A}}$, and $\Delta_{\lambda}=\lambda_{O B U_{A}}-\lambda_{R S U_{A}}$.

We now have two GCs that intersect at point $R S U_{A}$, as required to determine the angular distances $R_{\Delta}^{A}$ and $L_{\Delta}^{A}$, as shown in Fig. 7. Note that these projections over the respective GCs have a sign rule, given by the position of 365 the point with respect to the GC. For example, if the point is on the right side of $\mathrm{GC}\left(R S U_{A}, R_{A}\right)$, like $O B U_{A}$, then $R_{\Delta}^{A}$ is negative. Otherwise, it is positive. The same occurs for $\operatorname{GC}\left(R S U_{B}, R_{B}\right)$.

Denoting the Azimuth $\beta_{R A}=\beta_{A B}+\pi / 2$, the angular distance $R_{\Delta}^{A}$ from point $O B U_{A}$ at $\left(\phi_{O B U_{A}}, \lambda_{O B U_{A}}\right)$ to $\operatorname{GC}\left(R S U_{A}, R_{A}\right)$ can be calculated, given 370 the initial azimuth $\beta_{A A}$, the angular distance $d_{A A}$, and the initial azimuth $\beta_{R A}$, using spherical trigonometry [36], as:

$$
\left.R_{\Delta}^{A}=\arcsin \left(d_{A A}\right) \cdot \sin \left(\beta_{A A}-\beta_{R A}\right)\right) .
$$

Similarly, the angular distance $L_{\Delta}^{A}$ from point $\mathrm{O} B U_{A}$ at $\left(\phi_{O B U_{A}}, \lambda_{O B U_{A}}\right)$ to the $\operatorname{GC}\left(R S U_{A}, R S U_{B}\right)$ can be calculated, given the initial Azimuth $\beta_{A A}$, the angular distance $d_{A A}$, and the initial Azimuth $\beta_{A B}$, as:

$$
\left.L_{\Delta}^{A}=\arcsin \left(d_{A A}\right) \cdot \sin \left(\beta_{A A}-\beta_{A B}\right)\right) .
$$




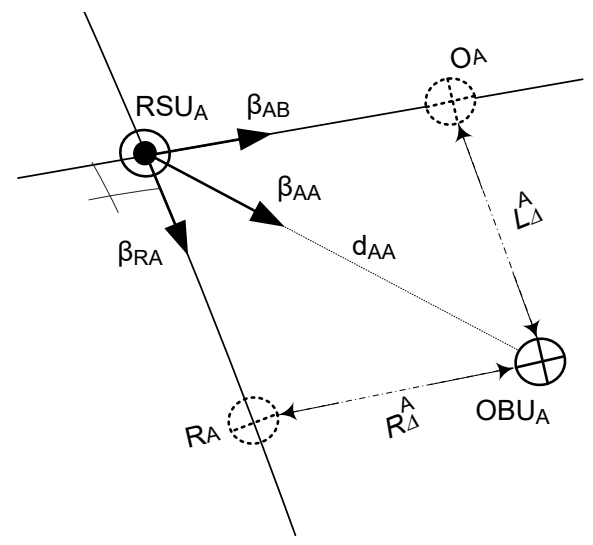

Figure 7: Calculation of the projections $R_{\Delta}$ and $L_{\Delta}$ over the $\operatorname{GC}\left(R S U_{A}, R S U_{B}\right)$ and $\mathrm{GC}\left(R S U_{A}, R_{A}\right)$ using spherical trigonometric relations. $R_{\Delta}$ and $L_{\Delta}$ are function of distance and bearing between $R S U_{A}$ and $O B U_{A}$ and bearing of the corresponding GC projections.

$R_{\Delta}$ and $L_{\Delta}$ are calculated for every new position acquired from the GPS receiver and correspond to the distances to the respective GCs, the fixed references. These distances can be broadcast through Basic Safety Messages (BSMs) [37. to quickly estimate collision probability.

\subsection{Considering the GPS Update Rate}

Each GPS device has an update rate which defines the frequency new data is sent to the user application. Even for high precision devices, where the update period is $100 \mathrm{~ms}$, when the vehicle drives at speeds above $120 \mathrm{~km} / \mathrm{h}$, the distance traveled during this refresh period is greater than $3 \mathrm{~m}$. As this distance can compromise the accuracy of vehicle safety applications, we use a simple method derived from the CooPS geometry model to improve GPS accuracy.

Denoting $t_{0}$ as the time of the last GPS update, $\left(\phi_{0}, \lambda_{0}\right)$ the last coordinates, $v_{0}$ the last vehicle speed, the arc $r$ traveled during the update interval $T_{\text {update }}$ is:

$$
r=\left(t-t_{0}\right) \cdot v_{0}, 0 \leq t-t_{0} \leq T_{\text {update }}
$$


where $t$ is the current time. Denoting $h_{0}$ the last heading angle the latitude $\phi_{c}$ during this interval can be calculated as:

$$
\phi_{c}=\operatorname{asin}\left(\sin \left(\phi_{0}\right) \cdot \cos (r)+\cos \left(\phi_{0}\right) \cdot \sin (r) \cdot \cos \left(h_{0}\right)\right) .
$$

Defining $a=\sin \left(h_{0}\right) \cdot \sin (r) \cdot \cos \left(\phi_{0}\right)$ and $b=\cos (r)-\sin \left(\phi_{0}\right) \cdot \sin \left(\phi_{c}\right)$, the longitude $\lambda_{c}$ during the $T_{\text {update }}$ is:

$$
\lambda_{c}=\lambda_{0}+\operatorname{atan} 2(a, b) .
$$

Thus, the safety application does not need to wait the next update. Instead, it can call this algorithm to calculate the current coordinates $\left(\phi_{c}, \lambda_{c}\right)$, improving GPS accuracy.

\section{Performance Evaluation}

We evaluate the performance of CooPS through real experiments conducted at a two-way street in the campus of UFRJ. Fig. 8 shows the experimental scenario. All GPS receivers are in line-of-sight conditions. Also, the GPS receivers share the same environment and there are no tall buildings or trees within the experiment perimeter. The two RSUs are installed at a height of $1.5 \mathrm{~m}$, separated by a ground distance of $407.64 \mathrm{~m}$. The geographic coordinates of the

RSUs ( $\phi=$ Latitude, $\lambda=$ Longitude) were extracted from landmarks on the Google Earth map (a vertical white line for $R S U_{A}$ and a light pole for $R S U_{B}$ ), their values are:

$$
\begin{aligned}
& R S U_{A}: \quad\left(\phi_{R S U_{A}}=-22.862084, \lambda_{R S U_{A}}=-43.22487\right) \\
& R S U_{B}: \quad\left(\phi_{R S U_{B}}=-22.860038, \lambda_{R S U_{B}}=-43.221572\right) .
\end{aligned}
$$

To evaluate the accuracy of CooPS regarding to the road axis, Lanes $1 \mathrm{~A}$ and $1 \mathrm{~B}$ were used whereas for the lane axis, Lanes $1 \mathrm{~A}$ and 2 were used (Fig. 8). The distances from the RSUs to the center of Lanes 1A, 1B, and 2 are 2.68, 12.60 , and $6.20 \mathrm{~m}$, respectively. A vehicle with an embedded OBU traveled 15 times on Lane 1A, 15 times on Lane 2, and 30 times on Lane 1B at speeds 


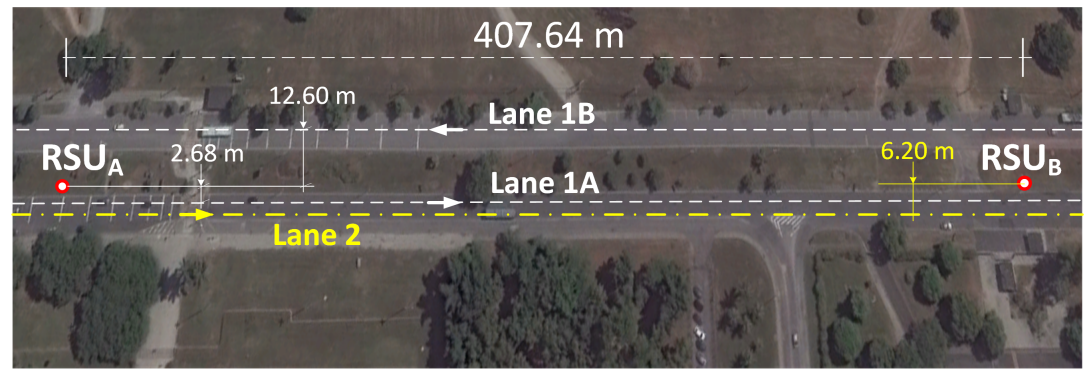

Figure 8: Experimental scenario used for CooPS evaluation performance. Three separate road stretches were used, Lanes $1 \mathrm{~A}, 1 \mathrm{~B}$, and 2 . We used two fixed road-side units: $R S U_{A}$ and $R S U_{B}$ (figure produced using Google Earth).

between 20 and $60 \mathrm{~km} / \mathrm{h}$. The set of hardware used in the experiments is listed in Table 2. The CooPS elliptical geometry model was implemented using $\mathrm{Ge}$ ographicLib [38. The RSUs and OBUs are equipped with single-carrier, $2.5 \mathrm{~m}$ accuracy CEP of $50 \%$ GPS receivers operating at $5 \mathrm{~Hz}$ navigation update rate. They are also equipped with two IEEE 802.11 p radios used for V2V and V2I communications over the DSRC band, working at the power level of $23 \mathrm{dBm}$. Basic safety Messages between RSUs and OBU were sent on DSRC channel 178.

Table 2: Equipment used in the experiments.

\begin{tabular}{|l|c|}
\hline Hardware & Description \\
\hline \hline RSU & Cohda Wireless model MK5-RSU \\
\hline OBU & Cohda Wireless model MK5-OBU \\
\hline DSRC Antenna & $2 \times 5.9$ GHz MobileMarkECO6-5500e \\
\hline GNSS Antenna & $1 \times$ WELL-HOPE GPS/GLON-09B \\
\hline Vehicle & 2015 Peugeot 408 \\
\hline
\end{tabular}

\subsection{Results}

In our experiments, we collect the values of $R_{\Delta}, L_{\Delta}$ every time the vehicle travels the road stretch from $R S U_{A}$ to $R S U_{B}$ and from $R S U_{B}$ to $R S U_{A}$. During that time, the vehicle speed and coordinates provided by the GPS receiver 
embedded in the OBU are also collected. To evaluate the precision of CooPS to estimate the relative distance to the road axis, we compute the values of $R_{\Delta}$ taking the vehicle traveling direction into account. When the vehicle goes from $R S U_{A}$ to $R S U_{B}$ (Lane $1 \mathrm{~A}$ ), $R_{\Delta}$ is computed from the $R S U_{A}$ coordinates, until the vehicle overpasses $R S U_{B}$. This event is detected at the moment the vehicle crosses $\mathrm{GC}\left(R S U_{B}, R_{B}\right)$, as shown in Fig. 6. Similarly for Lane 1B, $R_{\Delta}$ is computed from the $R S U_{B}$ coordinates until the vehicle overpasses $R S U_{A}$.

These values are compared with the distance between RSUs, calculated using absolute coordinates. Nevertheless, due to the GPS update rate combined with the vehicle speed, the signal changing detection of $R_{\Delta}$ happens after a random time interval, resulting in an additional distance, $d_{c r}$, given by:

$$
d_{c r}=v_{c r} \cdot t_{c r}
$$

where $v_{c r}$ is the vehicle speed when it crosses the GC and $t_{c r}$ is a random fraction of the GPS update period. Assuming that $t_{c r}$ is a random discrete variable with uniform distribution over [0,200 ms] interval, where $200 \mathrm{~ms}$ is the update period of the used GPS, the expected value of $d_{c r}$ is:

$$
E\left(d_{c r}\right)=E\left(v_{c r}\right) \cdot E\left(t_{c r}\right)
$$

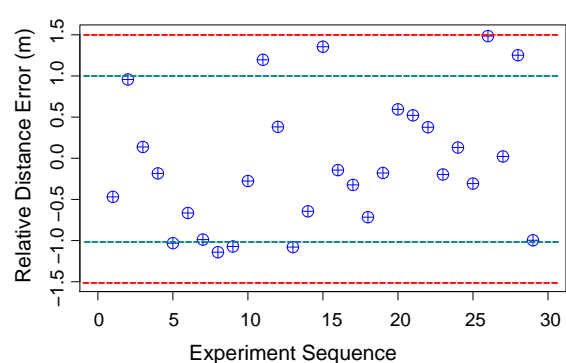

(a) Lane 1A Relative Distance Error.

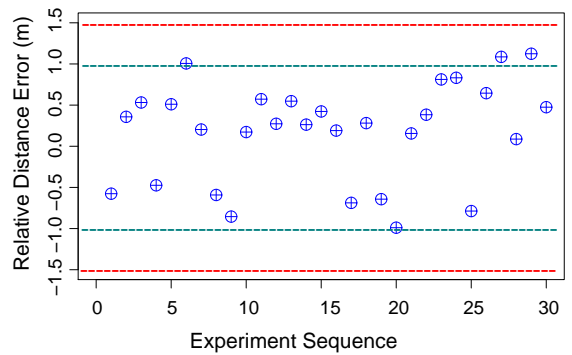

(b) Lane 1B Relative Distance Error.

Figure 9: CooPS Road Axis Performance to estimate the distance between $R S U_{A}$ and $R S U_{B}$. The dotted red lines denote which-lane and green ones where-in-lane boundaries 
Therefore, after extracting outliers and subtracting the corresponding expected values of $d_{c r}$, CooPS performance with respect to the road axis positioning error is shown in Fig. 9. Negative values mean an estimated distance shorter than the reference distance between the RSUs. The dotted red lines denote which-lane and green ones where-in-lane boundaries

Note that the performance of CooPS with respect to the road axis meets which-lane requirements. A better performance is observed for Lane 1B (Fig. 9b) which can be assigned by an average speed of experiment sequences lower than Lane 1A. This fact is confirmed by the numbers of Table 3 , which shows smaller standard deviation $(\sigma)$ and $95 \%$ confidence interval for the experiments over Lane 1B.

Table 3: Statistical data of road axis CooPS relative error acquired when the vehicle crosses the great circles.

\begin{tabular}{cccc}
\cline { 2 - 4 } Lane & \multicolumn{3}{c}{ Road Axis Relative Error } \\
\cline { 2 - 4 } & Mean $(\mathrm{m})$ & $\sigma(\mathrm{m})$ & Conf. Int. $(\mathrm{m})$ \\
\hline 1A & -0.06 & 0.78 & {$[-0.36,0.23]$} \\
1B & 0.17 & 0.60 & {$[-0.04,0.40]$} \\
\hline
\end{tabular}

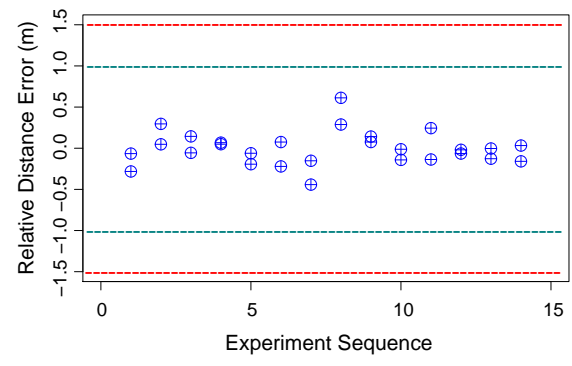

(a) Lane 1A.

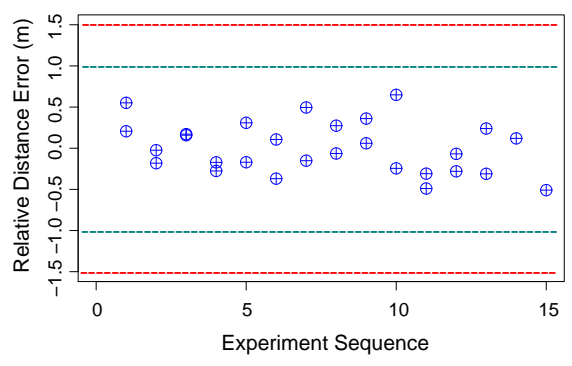

(b) Lane 2.

Figure 10: CooPS Lane Axis Error Evaluation.

Assuming a negligible lateral displacement of the vehicle during the experiments, CooPS performance evaluation with respect to the lane axis was per- 

confirmed by the statistical data of Table 4. These values mean that CooPS exceeds which-lane requirements and provides where-in-lane accuracy level.

Table 4: Statistical data of lane axis CooPS relative error acquired when the vehicle crosses the great circles.

\begin{tabular}{lccc}
\cline { 2 - 4 } Lane & \multicolumn{3}{c}{ Lane Axis Relative Error } \\
\cline { 2 - 4 } & Mean $(\mathrm{m})$ & $\sigma(\mathrm{m})$ & Conf. Int. $(\mathrm{m})$ \\
\hline 1A & 0.00 & 0.21 & {$[-0.08,0.07]$} \\
2 & 0.00 & 0.31 & {$[-0.11,0.12]$} \\
\hline
\end{tabular}

\subsection{Validation}

We validate the results plotting the $R_{\Delta}$ and $L_{\Delta}$ projections calculated by CooPS on Google Earth map in two scenarios. In the first one, we compare map ground position with CooPS estimated absolute position $\left(R_{\Delta}\right)$ from the $R S U_{A}$ to points around 100, 200, 300 and $400 \mathrm{~m}$ along road stretch acquired when the vehicle traveled on Lane 1A (Fig. 11). We compare 60 points corresponding to 15 passages through 4 landmarks. The statistics shown in Table 5 reveal a similar behavior among landmarks position errors and confirm CooPS accuracy stability along the stretch limited by $R S U_{A}$ and $R S U_{B}$.

In the second validation scenario, we compare CooPS position estimation with Google Earth map ground position when the vehicle crosses the great circles $\operatorname{GC}\left(R S U_{A}, R_{A}\right)$ and $\operatorname{GC}\left(R S U_{B}, R_{B}\right)$. We illustrate the results using the Google Earth map, as shown in Fig. 12. Figs. 12a and 12b are the zoomed area of the rectangle depicted in their upper left corners. The projections correspond 
Table 5: Statistical data of position errors acquired by the comparison between CooPS absolute position estimation and Google Earth map ground position of the distances from $R S U_{A}$ to the landmarks at $100,200,300$ and $400 \mathrm{~m}$.

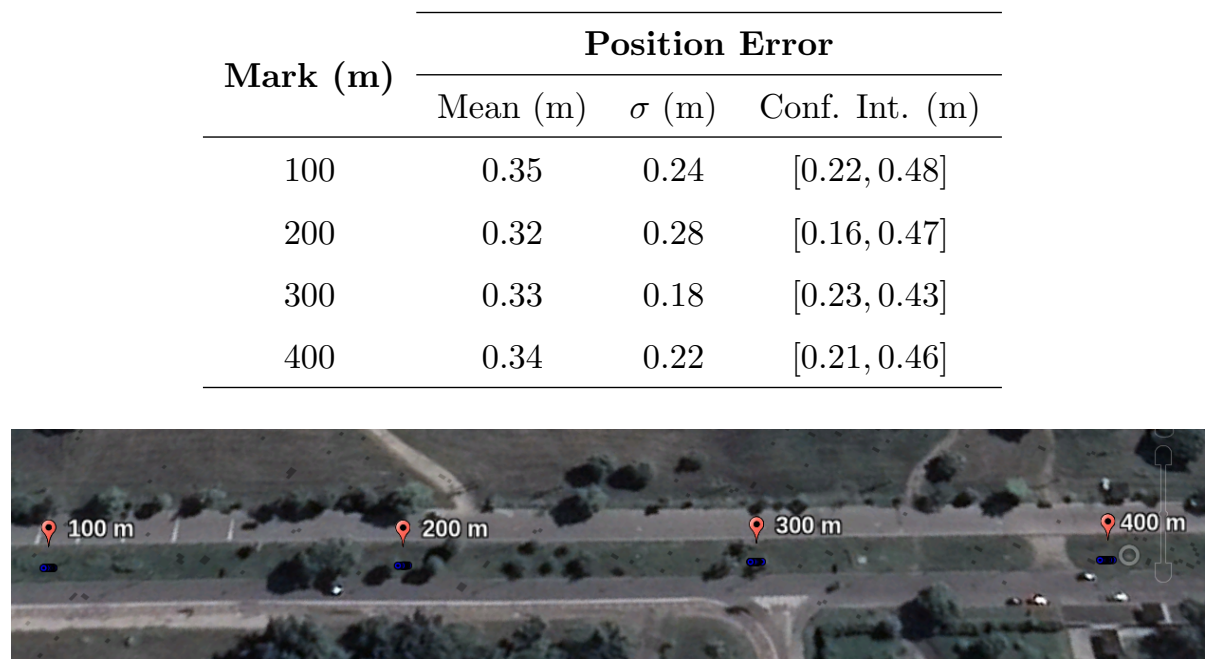

Figure 11: CooPS absolute position validation. The landmarks 100, 200, 300 and $400 \mathrm{~m}$ are the corresponding ground positions from $R S U_{A}$. The errors are acquired from the comparison between CooPS estimation positions and Google Earth map positions (figure produced using Google Earth).
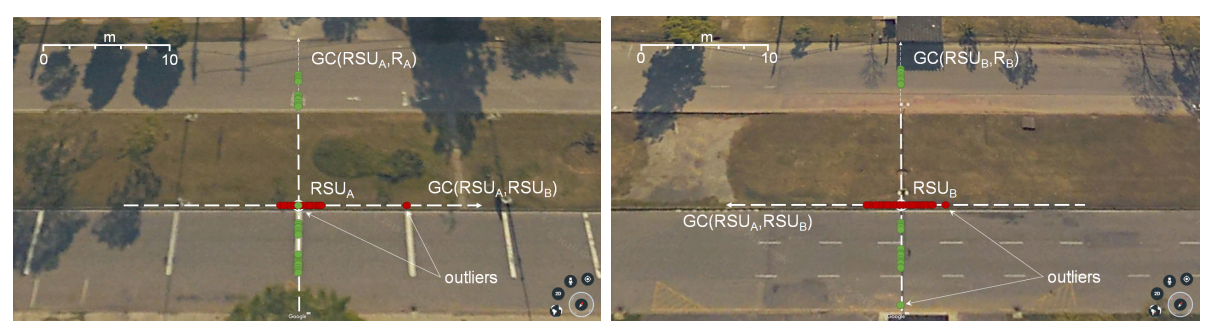

(a) Great Circle $\mathrm{GC}\left(R S U_{A}, R_{A}\right)$ crossing. (b) Great Circle $\operatorname{GC}\left(R S U_{B}, R_{B}\right)$ crossing.

Figure 12: CooPS great circle crossing validation. The red points are the coordinates of $R_{\Delta}$ projections, green ones are the coordinates of $L_{\Delta}$ projections over $\operatorname{GC}\left(R S U_{A}, R_{A}\right)$ and $\operatorname{GC}\left(R S U_{B}, R_{B}\right)$ (white dashed lines). These points are acquired when the vehicle crosses the great circles $\operatorname{GC}\left(R S U_{A}, R_{A}\right)$ and $\operatorname{GC}\left(R S U_{B}, R_{B}\right)$ corresponding to Figs. $12 \mathrm{a}$ and $12 \mathrm{~b}$ respectively. 
to the nearest positions of the vehicle to the GCs in both Lane 1 (A\&B) and Lane 2. The accuracy related to Road Axis (red dots) and related to the Lane Axis (green dots) can be evaluated comparing with the map scale. The figure also shows the outliers removed from the experiment's data.

\section{Concluding Remarks}

This work presented CooPS, a cooperative positioning system that meets the accuracy requirements of vehicle safety applications. To achieve these requirements, CooPS employs IEEE 802.11p V2I and V2V communications, in a cooperation between vehicles and RSUs. CooPS uses differential GNSS through position vector differencing to compute the relative distance between vehicles and the surveyed-coordinates RSUs. The development of CooPS involved the analysis of GNSS receiver error sources and an experimental evaluation campaign where consecutive position errors over 24 hours from two static GPS receivers were collected. CooPS has proved that the multipath error behavior of roving GPS receivers is similar to stationary ones in the vehicular environment if all the receivers share the same satellite constellation and ephemerids and considering the short time during which vehicle interactions occur. CooPS also includes simple methods to handle the determination of relative and absolute position and to improve accuracy between updates. The system performance is confirmed by the field experiment results, using off-the-shelf IEEE 802.11p OBUs and RSUs. The results have shown a relative distance accuracy level under $1.5 \mathrm{~m}$ with respect to the road axis and under $1.0 \mathrm{~m}$ with respect to the lane axis. Despite the adoption of a single carrier GNSS as the unique positioning device, CooPS was able to provide positioning accuracy sufficient to deploy safety applications in vehicular environments, providing low cost and ease of installation, a step further with respect to state-of-art systems.

As future work we will develop a dead-reckoning subsystem to enable CooPS to use vehicle factory assembled sensors data to overcome GNSS unreliable data and unavailability due to urban canyons, dense forest canopies, and tunnels. 


\section{Acknowledgements}

We are thankful to the Applied Superconductivity Laboratory Team for their support. This work was partially funded by FAPERJ, CNPq, CAPES, and do Estado de São Paulo (FAPESP).

\section{References}

[1] N. Alam, A. G. Dempster, Cooperative positioning for vehicular networks: Facts and future, IEEE Transactions on Intelligent Transportation Systems 14 (4) (2013) 1708-1717. doi:10.1109/TITS.2013.2266339

[2] N. Lu, N. Cheng, N. Zhang, X. Shen, J. W. Mark, Connected vehicles: Solutions and challenges, IEEE Internet of Things Journal 1 (4) (2014) 289-299. doi:10.1109/JIOT.2014.2327587.

[3] E. Stenborg, L. Hammarstrand, Using a single band GNSS receiver to im-

[5] M. Abbas, M. Karsiti, M. Napiah, B. Samir, M. Al-Jemeli, High accuracy traffic light controller for increasing the given green time utilization, Computers \& Electrical Engineering 41 (2015) 40-51. doi:https: //doi.org/10.1016/j.compeleceng.2014.12.011

525

[6] F. Aloul, I. Zualkernan, R. Abu-Salma, H. Al-Ali, M. Al-Merri, iBump: Smartphone application to detect car accidents, Computers \& Electri口 cal Engineering 43 (2015) 66-75. compeleceng.2015.03.003. 
[7] J. Liu, B. Cai, Y. Wang, J. Wang, A lane level positioning-based coop-

[9] P. Sathiya, P. Anandhakumar, Probabilistic collision estimation for tracked vehicles based on corner point self-activation approach, Computers \& Electrical Engineering. doi:https://doi.org/10.1016/j. compeleceng.2018.07.029.

[10] F. Riaz, S. Jabbar, M. Sajid, M. Ahmad, K. Naseer, N. Ali, A collision avoidance scheme for autonomous vehicles inspired by human social norms,

n Computers \& Electrical Engineering 69 (2018) 690 - 704. doi:https:

[11] S. Zair, S. L. Hégarat-Mascle, E. Seignez, A-contrario modeling for robust localization using raw GNSS data, IEEE Transactions on Intelligent Trans-

[12] A. Lambert, D. Gruyer, G. S. Pierre, A. N. Ndjeng, Collision probability assessment for speed control, in: 11th International IEEE Conference on 口 Intelligent Transportation Systems, 2008, pp. 1043-1048. doi:10.1109/ ITSC.2008.4732692.

[13] J. B. P. Neto, L. C. Gomes, E. M. Castanho, M. E. M. Campista, L. H. 555 M. K. Costa, P. C. M. Ribeiro, An error correction algorithm for forward collision warning applications, in: IEEE 19th International Conference on 
口 10.1109/ITSC. 2016.7795867,

[14] J. Zogg, GPS: Essentials of Satellite Navigation : Compendium : Theory and Principles of Satellite Navigation, Overview of GPS/GNSS Systems and Applications, U-blox AG, 2009.

[15] K. Wisiol, M. Wieser, R. Lesjak, GNSS-based vehicle state determination tailored to cooperative driving and collision avoidance, in: European Navigation Conference (ENC), 2016, pp. 1-8. doi:10.1109/EURONAV.2016. 7530562

[16] K. Jo, Y. Jo, J. K. Suhr, H. G. Jung, M. Sunwoo, Precise localization of an autonomous car based on probabilistic noise models of road surface marker features using multiple cameras, IEEE Transactions on Intelligent Transportation Systems 16 (6) (2015) 3377-3392.

[17] A. Y. Hata, D. F. Wolf, Feature detection for vehicle localization in urban environments using a multilayer LIDAR, IEEE Transactions on Intelligent Transportation Systems 17 (2) (2016) 420-429.

[18] A. Scheel, C. Knill, S. Reuter, K. Dietmayer, Multi-sensor multi-object tracking of vehicles using high-resolution radars, in: IEEE Intelligent Vehicles Symposium (IV), 2016, pp. 558-565.

[19] T. Williams, P. Alves, G. Lachapelle, C. Basnayake, Evaluation of GPSbased methods of relative positioning for automotive safety applications, Transportation Research Part C: Emerging Technologies 23 (2012) 98 108. doi:http://dx.doi.org/10.1016/j.trc.2011.08.011.

[20] M. Shao, X. Sui, Study on differential GPS positioning methods, in: International Conference on Computer Science and Mechanical Automation (CSMA), 2015, pp. 223-225. doi:10.1109/CSMA.2015.51. 
[21] J. K. Suhr, J. Jang, D. Min, H. G. Jung, Sensor fusion-based low-cost vehicle localization system for complex urban environments, IEEE Transactions on Intelligent Transportation Systems 18 (5) (2017) 1078-1086.

[22] M.-F. Tsai, P.-C. Wang, C.-K. Shieh, W.-S. Hwang, N. Chilamkurti, S. Rho, Y. S. Lee, Improving positioning accuracy for VANET in real city environments, The Journal of Supercomputing 71 (6) (2015) 1975-1995.

[23] K. Ansari, C. Wang, L. Wang, Y. Feng, Vehicle-to-vehicle real-time relative positioning using $5.9 \mathrm{GHz}$ DSRC media, in: IEEE Vehicular Technology Conference (VTC Fall), 2013, pp. 1-7.

[24] R. M. Kalafus, A. Van Dierendonck, N. A. Pealer, Special committee 104 recommendations for differential GPS service, Navigation 33 (1) (1986) $26-41$.

[25] Z. He, W. Tang, X. Yang, L. Wang, J. Liu, Use of ntrip for optimizing the decoding algorithm for real-time data streams, Sensors 14 (10) (2014) 18878-18885. doi:10.3390/s141018878.

URL http://www.mdpi .com/1424-8220/14/10/18878

[26] J. Roth, T. Schaich, G. F. Trommer, Cooperative GNSS-based method for vehicle positioning, Gyroscopy and Navigation 3 (4) (2012) 245-254.

[27] C. M. Huang, S. Y. Lin, Cooperative vehicle collision warning system using the vector-based approach with dedicated short range communication data transmission, IET Intelligent Transport Systems 8 (2) (2014) 124-134. doi : 10.1049/iet-its.2012.0101

[28] R. Karlsson, F. Gustafsson, The future of automotive localization algorithms: Available, reliable, and scalable localization: Anywhere and ロ anytime, IEEE Signal Processing Magazine 34 (2) (2017) 60-69. doi: 10.1109/MSP. 2016.2637418

[29] K. Lassoued, P. Bonnifait, I. Fantoni, Cooperative localization of vehicles sharing GNSS pseudoranges corrections with no base station using set in- 
version, in: IEEE Intelligent Vehicles Symposium (IV), 2016, pp. 496-501. doi:10.1109/IVS.2016.7535432.

[30] M. S. Grewal, A. P. Andrews, C. G. Bartone, Global Navigation Satellite Systems, Inertial Navigation, and Integration, 3rd Edition, WileyInterscience, New York, NY, USA, 2013.

[31] S. Demmel, A. Lambert, D. Gruyer, A. Rakotonirainy, E. Monacelli, Empirical IEEE 802.11p performance evaluation on test tracks, in: IEEE Intelligent Vehicles Symposium, 2012, pp. 837-842.

[32] F. A. Teixeira, V. F. e Silva, J. L. Leoni, D. F. Macedo, J. M. Nogueira, Vehicular networks using the IEEE 802.11p standard, Vehicular Communications 1 (2) (2014) 91-96. doi:10.1016/j.vehcom.2014.04.001.

[33] T. Vincenty, Direct and inverse solutions of geodesics on the ellipsoid with applications of nested equations, Survey Review 23 (176) (1975) 88-93.

[34] C. F. F. Karney, Algorithms for geodesics, Journal of Geodesy 87 (1) (2013) $43-55$.

[35] F. Ivis, Calculating geographic distance: Concepts and Methods, 2006, Canadian Institute for Health Information (2006).

[36] C. Veness, Movable Type Scripts (2016 (accessed October 10, 2018)). URL http://williams . best.vwh.net/avform.htm

[37] W. Liu, B. Li, Research on the Mechanism of Intelligent Transportation Systems on Improving Road Safety, Springer Berlin Heidelberg, Berlin, Heidelberg, 2015, Ch. 26, pp. 257-263.

[38] C. Karney, Geographiclib (2015 (accessed October 10, 2018)). URL https://geographiclib.sourceforge.io/ 